\title{
STABILITY RESULTS FOR SOME NONLINEAR ELLIPTIC EQUATIONS INVOLVING THE $p$-LAPLACIAN WITH CRITICAL SOBOLEV GROWTH
}

\author{
Bruno NAZARET ${ }^{1,2}$
}

\begin{abstract}
This article is devoted to the study of a perturbation with a viscosity term in an elliptic equation involving the $p$-Laplacian operator and related to the best contant problem in Sobolev inequalities in the critical case. We prove first that this problem, together with the equation, is stable under this perturbation, assuming some conditions on the datas. In the next section, we show that the zero solution is strongly isolated in some sense, among the space of the solutions. Actually, we end the paper by giving some analoguous results in the case where the datas present symmetries.

Résumé. Dans cet article, l'auteur se propose d'étudier une perturbation de type visqueux dans une équation elliptique contenant l'opérateur du $p$-Laplacien, et provenant d'un problème de meilleure constante pour les inégalités de Sobolev dans le cas critique. Nous montrons ici que ce problème, ainsi que l'équation associée est stable sous cette perturbation, moyennant quelques hypothèses sur les données. Dans la suite, nous prouvons que la solution identiquement nulle est, dans un certain sens, isolée dans l'espace des solutions. Enfin, nous terminons cette étude par des résultats analogues dans le cas où les données possèdent des symétries.
\end{abstract}

AMS Subject Classification. 35J20, 35J60, 35J70, 49K20.

Received July 10, 1998. Revised December 4, 1998.

\section{INTRODUCTION}

In this paper, we are interested in the stability under a perturbation with a viscosity term of the following nonlinear elliptic PDE's involving the $p$-Laplacian with critical Sobolev growth:

$$
\left\{\begin{array}{l}
-\operatorname{div}\left(|\nabla u|^{p-2} \nabla u\right)+a(x)|u|^{p-2} u=f(x)|u|^{p^{*}-2} u \\
u \in W_{0}^{1, p}(\Omega) .
\end{array}\right.
$$

Here, $\Omega$ denotes a bounded open set of $\mathbb{R}^{N}, a$ and $f$ are smooth on $\bar{\Omega}, p$ is a real in $(1, N)$, and $p^{*}=N p /(N-p)$ is the critical exponent for the Sobolev embedding of $W_{0}^{1, p}(\Omega)$ in $L^{q}(\Omega)$.

Keywords and phrases: Variational problems, nonlinear elliptic PDE, optimal Sobolev inequalities, best constant problems, $p$-Laplacian operator.

${ }^{1}$ ENS Cachan, Antenne de Bretagne, Campus de Ker Lann, 35170 Bruz, France.

${ }^{2}$ Université de Cergy-Pontoise, Département de Mathématiques, 2 avenue Adolphe Chauvin, 95302 Cergy-Pontoise, France.

(c) EDP Sciences, SMAI 1999 
When $a$ is constant and $f \equiv 1$, equation (1) is the Euler equation of the following minimisation problem:

$$
\lambda(N, p, \Omega)=\inf _{u \in W_{0}^{1, p}(\Omega), u \neq 0} \frac{\int_{\Omega}\left(|\nabla u|^{p}+a|u|^{p}\right)}{\left(\int_{\Omega}|u|^{p^{*}}\right)^{\frac{p}{p^{*}}}} .
$$

In other words, $\lambda(N, p, \Omega)^{-1 / p}$ is the first best constant for the embedding of $W_{0}^{1, p}(\Omega)$ in $L^{p^{*}}(\Omega)$ (see Hebey [11]). In the case where $\Omega=\mathbb{R}^{N}$, the supremum defined by:

$$
K(N, p)=\sup _{u \in C_{0}^{\infty}\left(\mathbb{R}^{N}\right)} \frac{\left(\int_{\mathbb{R}^{N}}|u|^{p^{*}}\right)^{\frac{1}{p^{*}}}}{\left(\int_{\mathbb{R}^{N}}|\nabla u|^{p}\right)^{\frac{1}{p}}}
$$

has been computed by Aubin [1] and Talenti [16], and has value:

$$
K(N, p)=\frac{p-1}{N-p}\left(\frac{N-p}{N(p-1)}\right)^{\frac{1}{p}}\left(\frac{\Gamma(N+1)}{\Gamma\left(\frac{N}{p}\right) \Gamma\left(N+1-\frac{N}{p}\right) \omega_{N-1}}\right)^{\frac{1}{N}}
$$

where $\omega_{N-1}$ denotes the volume of the unit sphere in $\mathbb{R}^{N}$. Furthermore, this supremum is achieved on the following functions:

$$
u_{\mu}(x)=\left(\mu+r^{\frac{p}{p-1}}\right)^{1-\frac{N}{p}}
$$

where $\mu$ is a parameter and $r$ the euclidean norm of $x$.

When $\Omega$ is arbitrary, Hebey [10], Hebey-Vaugon [8] (in the case where $p=2$ ), and Demengel-Hebey (in the case where $p>1$ ) study the existence of extremal functions (i.e. which realize the extremum) for problem (1) (see also Lions $[13,14])$ :

Let $a$ and $f$ be $C^{\infty}$ functions on $\bar{\Omega}$. We assume that $\Omega$ is bounded and regular. Then, we define

$$
\lambda(\Omega)=\inf _{u \in W_{0}^{1, p}(\Omega)} \frac{\int_{\Omega}\left(|\nabla u|^{p}+a(x)|u|^{p}\right)}{\left(\int_{\Omega} f(x)|u|^{p^{*}}\right)^{\frac{p}{p^{*}}}} .
$$

Note that, in this kind of problems, though the infimum value is in general not known, the case $\mathbb{R}^{N}$ acts as reference, since the authors above show that, if $\lambda(\Omega)<K(N, p)^{-p}\|f\|_{L^{\infty}}^{(p / N)-1}$, then this infimum is realized on a positive solution of the following equation:

$$
-\operatorname{div}\left(|\nabla u|^{p-2} \nabla u\right)+a(x)|u|^{p-2} u=\lambda(\Omega) f(x)|u|^{p^{*}-2} u .
$$

By regularity results, such as developped in Guedda-Veron $[6,7], u \in C^{1, \alpha}(\bar{\Omega})$, and by the Vazquez strict maximum principle [18], $u$ is positive in $\Omega$. They give also symmetry conditions on $\Omega$ and invariance conditions on $a$ and $f$, which imply the existence's condition $\lambda(\Omega)<K(N, p)^{-p}\|f\|_{L^{\infty}}^{p / N-1}$.

Our aim in this article is to show, for $p<2$ and under the condition above, that these solutions are stable under some viscosity perturbation. Furthermore, the proof of this result presents the advantage to give another proof of the existence theorem given by Demengel-Hebey.

Remark that, if $u$ is a solution of (3) and if $\int_{\Omega} f(x)|u|^{p^{*}}=1$, then $u$ realizes the infimum $\lambda(\Omega)$. Moreover, since the embedding of $W_{0}^{1, p}(\Omega)$ into $L^{p^{*}}(\Omega)$ is not compact, we can not solve equation (3) by standard variational 
arguments. In the perturbed equation, the viscosity term $-\epsilon \Delta u_{\epsilon}$ compensates the loss of compactness and give us a method in order to find a solution.

\section{Notations AND Results}

In this paper, $\Omega$ will denote a $C^{1}$ domain of $\mathbb{R}^{N}$, where $N \geq 3$. Let $p \in(1, N)$ be a real, and let $a, f$ be two $C^{\infty}$ functions defined on $\bar{\Omega}$. We are interested in the following problem:

$$
\left\{\begin{array}{l}
-\operatorname{div}\left(|\nabla u|^{p-2} \nabla u\right)+a(x)|u|^{p-2} u=f(x)|u|^{p^{*}-2} u \\
u \in W_{0}^{1, p}(\Omega)
\end{array}\right.
$$

where $p^{*}=\frac{N p}{N-p}$ is the critical Sobolev exponent for the embeddings $W^{1, p}(\Omega) \hookrightarrow L^{q}(\Omega)$.

We make the following assumptions:

- the function $a$ is such that the operator

$$
L(u)=-\operatorname{div}\left(|\nabla u|^{p-2} \nabla u\right)+a(x)|u|^{p-2} u
$$

is coercive, in the sense that there exists a positive constant $C$ such that, for all functions $u$ in $W_{0}^{1, p}(\Omega)$,

$$
J(u)=\int_{\Omega}\left(|\nabla u|^{p}+a(x)|u|^{p}\right) \geq C \int_{\Omega}|u|^{p} .
$$

- the function $f$ is positive somewhere in $\bar{\Omega}$ (this assumption is necessary, since $L$ is coercive).

Demengel and Hebey proved in [4] existence's results in the case where the data $\Omega, a$, and $f$ present some symmetries (for a Riemannian manifold, this problem is treated by Druet in [3]). Since we are not interested here in finding concrete conditions for the existence of extremal functions, we only considere the general case. (We make at the end of the paper a brief study of the presence of symmetries.) Then, the result of [4] can be written in the following simplified form: let us define the set

$$
\mathcal{W}_{p^{*}}=\left\{v \in W_{0}^{1, p}(\Omega) ; \int_{\Omega} f(x)|v|^{p^{*}}=1\right\}
$$

Theorem 2.1. Let us suppose that, for all $x \in \bar{\Omega}$ such that $f(x)>0$, we have:

$$
K(N, p)^{p} \lambda f(x)^{1-p / N}<1
$$

where

$$
\lambda=\inf _{v \in \mathcal{W}_{p^{*}}} J(v) .
$$

Then, there exists a solution $u$ of (4) in $W_{0}^{1, p}(\Omega) \cap C^{1, \alpha}(\bar{\Omega}), \alpha \in(0,1)$, which is positive. Moreover, $u$ realizes the infimum in (5).

In the first section, we shall prove, in the case where $p<2$, a stability result of the positive solutions of equation (4) under some perturbation by a viscous term. More precisely, we consider the variational problem:

$$
\lambda_{\epsilon}=\inf _{\int_{\Omega} f|v|^{p^{*}}=1}\left(\frac{\epsilon}{2} \int_{\Omega}|\nabla v|^{2}+J(v)\right)
$$


where $\epsilon$ is a positive real, which will tend to 0 later. Since $p<2, p^{*}$ is subcritical for the embedding of $H_{0}^{1}(\Omega)$ in $L^{q}(\Omega)$, and then, by standard compactness arguments, this problem admits a non zero solution $u_{\epsilon}$. In addition, $u_{\epsilon}$ solves the following equation:

$$
-\frac{\epsilon}{p} \Delta u_{\epsilon}-\operatorname{div}\left(\left|\nabla u_{\epsilon}\right|^{p-2} \nabla u_{\epsilon}\right)+a(x)\left|u_{\epsilon}\right|^{p-2} u_{\epsilon}=\mu_{\epsilon} f(x)\left|u_{\epsilon}\right|^{p^{*}-2} u_{\epsilon}
$$

where $\mu_{\epsilon}$ is some Lagrange multiplier.

Then, we prove the following result:

Theorem 2.2. We suppose that the assumptions in Theorem 2.1 hold. Let $u_{\epsilon}$ be a solution of (6), which is positive. Then, up to a subsequence $\left(u_{\epsilon}\right)$ converges strongly in $W_{0}^{1, p}(\Omega)$ to a solution $u$ of (4) which belongs to $W_{0}^{1, p}(\Omega) \cap C^{1, \alpha}(\bar{\Omega}), \alpha \in(0,1)$, is positive in $\Omega$, and realizes the infimum in (5).

In the next section, we are interested in the weak continuity of the set of solutions for equation (4) and we prove the following theorem:

Theorem 2.3. Let $\left(u_{n}\right)$ be a bounded sequence (in $W_{0}^{1, p}(\Omega)$ ) of solutions of (4) which are non identically zero. We assume that, at every point $x$ in $\bar{\Omega}$ where $f(x)>0$,

$$
K(N, p)^{p} \lambda f(x)^{1-p / N}<1
$$

and that

$$
\lim _{n \rightarrow \infty} \int_{\Omega} f(x)\left|u_{n}\right|^{p^{*}}=\lambda^{N / p}
$$

Then, $\left(u_{n}\right)$ converges strongly in $W_{0}^{1, p}(\Omega)$ to a nonzero solution $u$ of (4).

Finally, in the last section, we present a brief discussion concerning the case where the domain $\Omega$ is invariant under the action of a subgroup $G$ of the orthogonal group $O_{N}\left(\mathbb{R}^{N}\right)$ and give stability results for positive and nodal solutions (we say nodal for a solution wich changes sign).

\section{Stability of Positive SOlutions}

Let us consider the following problem:

$$
\left\{\begin{array}{l}
-\operatorname{div}\left(|\nabla u|^{p-2} \nabla u\right)+a(x)|u|^{p-2} u=f(x)|u|^{p^{*}-2} u \text { in } \Omega \\
u=0 \text { on } \partial \Omega, u>0 \text { in } \Omega
\end{array}\right.
$$

where $p<2$.

This has been solved by Demengel-Hebey in [4] for every $p \in(1, N)$. Our goal here is to study its stability when the operator $L$ is perturbed by adding to it $-\epsilon \Delta$ and when $\epsilon$ goes to zero. Since $p<2$, the operator $L-\epsilon \Delta$ is smoothing $L$. We now introduce some notations:

- $\mathcal{H}_{p^{*}}=\left\{v \in H_{0}^{1}(\Omega) ; \int_{\Omega} f(x)|v|^{p^{*}}=1\right\}$.

- For $\epsilon \geq 0$, and $v \in H_{0}^{1}(\Omega)$,

$$
\begin{aligned}
J_{\epsilon}(v) & =\frac{\epsilon}{2} \int_{\Omega}|\nabla v|^{2}+\int_{\Omega}\left(|\nabla v|^{p}+a(x)|v|^{p}\right) \\
\lambda_{\epsilon} & =\inf _{v \in \mathcal{H}_{p^{*}}} J_{\epsilon}(v) .
\end{aligned}
$$

The functional $J_{\epsilon}$ represents the energy functional for the operator $-\epsilon \Delta+L$ and the real $\lambda_{\epsilon}$ is the minimal energy under the condition $\int_{\Omega} f|v|^{p^{*}}=1$ (we shall see later that the real $\lambda$ is also the minimal energy of the initial problem). 


\subsection{The perturbed problem}

Before studying the perturbed equation, let us note that:

Lemma 3.1. $\lambda=\lambda_{0}$

Proof. We obviously have $\lambda \leq \lambda_{0}$. To prove the reverse inequality, we first state that

$$
\overline{\mathcal{H}}_{p^{*}} W_{0}^{1, p}(\Omega)=\mathcal{W}_{p^{*}} .
$$

Indeed, let $v \in W_{0}^{1, p}(\Omega)$ such that $\int_{\Omega} f(x)|v|^{p^{*}}=1$. Then, there exists a sequence $\left(v_{n}\right)_{n \in \mathbb{N}}$ of functions in $H_{0}^{1}(\Omega)$ such that $v_{n}$ converges to $v$ in $W_{0}^{1, p}(\Omega)$, almost everywhere in $\Omega$ and, due to the Sobolev embedding theorem, in $L^{p^{*}}(\Omega)$. From this, we deduce that $\int_{\Omega} f(x)\left|v_{n}\right|^{p^{*}} \longrightarrow \int_{\Omega} f(x)|v|^{p^{*}}=1$. Defining, for $n$ large enough in order to have $\left(\int_{\Omega} f|u|^{p^{*}}\right)^{1 p^{*}}<\frac{1}{2}$,

$$
w_{n}=\frac{v_{n}}{\left(\int_{\Omega} f(x)\left|v_{n}\right|^{p^{*}}\right)^{\frac{1}{p^{*}}}}
$$

one clearly has $J_{0}\left(w_{n}\right) \longrightarrow J_{0}(v)$, i.e.

$$
\forall \eta>0, \exists N \in \mathbb{N}, n>N \Longrightarrow\left|J_{0}\left(w_{n}\right)-J_{0}(v)\right|<\eta,
$$

and consequently

$$
\lambda_{0} \leq J_{0}\left(w_{n}\right)<J_{0}(v)+\eta
$$

This inequality being true for arbitrary $v$ and $\eta$, the proof is completed. We now give the main result of this section.

Proposition 3.1. Let $\epsilon>0$ be given. Then, there exists $u_{\epsilon} \in \mathcal{H}_{p^{*}}, u_{\epsilon} \geq 0$ a.e. in $\Omega$, which is a solution of the minimization problem (11). Furthermore, $u_{\epsilon}$ is a weak solution of the equation:

$$
-\frac{\epsilon}{p} \Delta u_{\epsilon}-\operatorname{div}\left(\left|\nabla u_{\epsilon}\right|^{p-2} \nabla u_{\epsilon}\right)+a(x)\left|u_{\epsilon}\right|^{p-2} u_{\epsilon}=\mu_{\epsilon} f(x)\left|u_{\epsilon}\right|^{p^{*}-2} u_{\epsilon}
$$

where

$$
\mu_{\epsilon}=\lambda_{\epsilon}+\epsilon\left(\frac{1}{p}-\frac{1}{2}\right) \int_{\Omega}\left|\nabla u_{\epsilon}\right|^{2} .
$$

Proof. To prove the existence of $u_{\epsilon}$, let $\left(v_{n}\right)_{n \in \mathbb{N}}$ be a minimizing sequence for $J_{\epsilon}$. Since $|\nabla| v_{n}||=\left|\nabla v_{n}\right|$, one can assume that $v_{n}$ is nonnegative, for all $n \in \mathbb{N}$. Using the coercivity of $L$, one gets that $\left(v_{n}\right)$ is bounded in $H_{0}^{1}(\Omega)$. From Banach-Aloaglu and Rellich-Kondrakov theorems, there exists a subsequence, still denoted $\left(v_{n}\right)$, and a function $u_{\epsilon}$ in $H_{0}^{1}(\Omega)$, such that:

- $v_{n} \rightarrow u_{\epsilon}$ in $H_{0}^{1}(\Omega)$ (and then in $W_{0}^{1, p}(\Omega)$ );

- $v_{n} \longrightarrow u_{\epsilon}$ in $L^{p^{*}}(\Omega)$;

- $v_{n} \longrightarrow u_{\epsilon}$ a.e. in $\Omega$;

where $u_{\epsilon} \in H_{0}^{1}(\Omega)$.

By the last two assertions, one gets that $u_{\epsilon} \geq 0$ a.e. in $\Omega$, and that $\int_{\Omega} f\left|u_{\epsilon}\right|^{p^{*}}=1$. By the lower semicontinuity of the norms in $W_{0}^{1, p}(\Omega)$ and $H_{0}^{1}(\Omega)$, one obtains

$$
J_{\epsilon}\left(u_{\epsilon}\right) \leq \lim \inf _{n \rightarrow \infty} J_{\epsilon}\left(v_{n}\right)=\lambda_{\epsilon}(G)
$$


and then $u_{\epsilon}$ solves (11). We now prove that $u_{\epsilon}$ is a weak solution of equation (12).

Let $v \in \mathcal{D}(\Omega)$ be given. Then, for every real $t$ small enough,

$$
J_{\epsilon}\left(\frac{u_{\epsilon}+t v}{\left(\int_{\Omega} f\left|u_{\epsilon}+t v\right|^{p^{*}}\right)^{\frac{1}{p^{*}}}}\right) \geq J_{\epsilon}\left(u_{\epsilon}\right) .
$$

By expanding the left hand side in powers of $t$ to the first order, one gets:

$$
\begin{aligned}
t\left(\frac{\epsilon}{p} \int_{\Omega} \nabla u_{\epsilon} \nabla v\right. & \left.+\int_{\Omega}\left|\nabla u_{\epsilon}\right|^{p-2} \nabla u_{\epsilon} \nabla v+\int_{\Omega} a(x)\left|u_{\epsilon}\right|^{p-2} u_{\epsilon} v\right) \geq t\left(\int_{\Omega} f(x)\left|u_{\epsilon}\right|^{p^{*}-2} u_{\epsilon} v\right) \\
& \times\left(\frac{\epsilon}{p} \int_{\Omega}\left|\nabla u_{\epsilon}\right|^{2}+\int_{\Omega}\left(\left|\nabla u_{\epsilon}\right|^{p}+a(x)\left|u_{\epsilon}\right|^{p}\right)\right)+\mathcal{O}\left(t^{2}\right) .
\end{aligned}
$$

It follows that for every function $v$ in $\mathcal{D}(\Omega)$,

$$
\frac{\epsilon}{p} \int_{\Omega} \nabla u_{\epsilon} \nabla v+\int_{\Omega}\left|\nabla u_{\epsilon}\right|^{p-2} \nabla u_{\epsilon} \nabla v+\int_{\Omega} a(x)\left|u_{\epsilon}\right|^{p-2} u_{\epsilon} v=\mu_{\epsilon} \int_{\Omega} f(x)\left|u_{\epsilon}\right|^{p^{*}-2} u_{\epsilon} v
$$

with $\mu_{\epsilon}=\lambda_{e}+\epsilon\left(\frac{1}{p}-\frac{1}{2}\right) \int_{\Omega}\left|\nabla u_{\epsilon}\right|^{2}$. This completes the proof.

In order to prove the stability of problem (4) under the perturbation defined in (7), we need some further results on the behaviour of the sequences $\left(u_{\epsilon}\right)$ and $\left(\mu_{\epsilon}\right)$.

Proposition 3.2. The sequence $\left(\lambda_{\epsilon}\right)$ tends to $\lambda$ as $\epsilon$ goes to 0 .

Proof. Let $\epsilon>\eta>0$ be given. Then, $\forall v \in \mathcal{H}_{p^{*}}, J_{\eta}(v)<J_{\epsilon}(v)$. Thus, $\lambda_{\eta} \leq \lambda_{\epsilon}$. We derive from this that $\left(\lambda_{\epsilon}\right)$ has a limit as $\epsilon$ goes to 0 . Moreover, by Lemma 3.1, this limit is greater than $\lambda$. Let us prove the reverse inequality.

Let $\delta>0$ be given. As we remarked in the proof of Lemma 3.1, there exists $v_{\delta} \in \mathcal{H}_{p^{*}}$ such that,

$$
\lambda \leq J_{0}\left(v_{\delta}\right) \leq \lambda+\delta
$$

Hence,

$$
\lambda_{\epsilon} \leq J_{\epsilon}\left(v_{\delta}\right)<\lambda+\delta+\frac{\epsilon}{2} \int_{\Omega}\left|\nabla v_{\delta}\right|^{2} .
$$

$\delta$ being arbitrary, we let $\epsilon$ go to 0 , and obtain

$$
\lim _{\epsilon \rightarrow 0} \lambda_{\epsilon} \leq \lambda+\delta
$$

which ends the proof.

Now, we give a strong convergence result concerning the perturbation term.

Proposition 3.3. Let $\left(u_{\epsilon}\right)$ be a sequence of solutions given by Proposition 3.1. Then, $\left(\sqrt{\epsilon} u_{\epsilon}\right)$ converges strongly to 0 in $H_{0}^{1}(\Omega)$.

Proof. For $\epsilon>\eta>0$ being given, one has

$$
\lambda_{\epsilon}-\lambda_{\eta} \geq J_{\epsilon}\left(u_{\epsilon}\right)-J_{\eta}\left(u_{\epsilon}\right) \geq \frac{\epsilon-\eta}{2} \int_{\Omega}\left|\nabla u_{\epsilon}\right|^{2} .
$$

By two repeated applications of Proposition 3.2 above, letting first $\eta$, and next $\epsilon$ go to 0 , one obtains the result. 
Corollary 3.4. The sequence $\left(\mu_{\epsilon}\right)$ tends to $\lambda$ as $\epsilon$ goes to 0 .

\subsection{Convergence of the perturbed problem}

We shall get a solution of (4) by extracting subsequences from the initial sequence $\left(u_{\epsilon}\right)_{\epsilon>0}$. By the way, the coercivity of the operator $L$, together with the convergence of $\left(\lambda_{\epsilon}\right)$, imply that the sequence $\left(u_{\epsilon}\right)$ is bounded in $W_{0}^{1, p}(\Omega)$. Then, up to a subsequence, it converges weakly in this space. The main difficulty here is to prove that the limit is not identically zero. This will be done in the next section.

Proposition 3.5. Let us suppose that there exists a subsequence of $\left(u_{\epsilon}\right)$ which converges weakly in $W_{0}^{1, p}(\Omega)$ to some function $u \not \equiv 0$. Then, $u$ belongs to $W_{0}^{1, p}(\Omega) \cap C^{1, \alpha}(\bar{\Omega})$, for all $\alpha \in(0,1)$, and $u$ is a solution of (4). Furthermore, $u$ realizes the infimum (5).

Proof. Up to a subsequence, one can assume that:

- $u_{\epsilon} \rightarrow u$ weakly in $W_{0}^{1, p}(\Omega)$;

- $u_{\epsilon} \longrightarrow u$ in $L^{k}(\Omega), \forall k<p^{*}$;

- $u_{\epsilon} \longrightarrow u$ a.e. in $\Omega$.

It turns out that $u \geq 0$ a.e. in $\Omega$.

In addition, the sequence $\left(\left|\nabla u_{\epsilon}\right|\right)$ is bounded in $L^{p}(\Omega)$, so $\left(\left|\nabla u_{\epsilon}\right|^{p-2} \nabla u_{\epsilon}\right)$ is bounded in $L^{p^{\prime}}(\Omega)$, where $p^{\prime}$ is the Hölder conjugate of $p$. Hence, there exists $\Sigma \in L^{p^{\prime}}(\Omega)$, such that, up to a subsequence,

- $\left|\nabla u_{\epsilon}\right|^{p-2} \nabla u_{\epsilon} \rightarrow \Sigma$, weakly in $L^{p^{\prime}}(\Omega)$.

Since $\left(\sqrt{\epsilon} u_{\epsilon}\right)$ converges strongly to 0 in $H_{0}^{1}(\Omega)$, passing to limit in (12), one obtains

$$
-\operatorname{div}(\Sigma)+a(x)|u|^{p-2} u=\lambda f(x)|u|^{p^{*}-2} u .
$$

Moreover, from (12), $\operatorname{div}\left(\epsilon \nabla u_{\epsilon}+\left|\nabla u_{\epsilon}\right|^{p-2} \nabla u_{\epsilon}\right)$ is bounded in $L^{\frac{p^{*}}{p^{*}-1}}(\Omega)$, and then in $L^{1}(\Omega)$. Using Lemma 3.2 below, one obtains that $\Sigma=|\nabla u|^{p-2} \nabla u$, and that $u$ is a solution of

$$
-\operatorname{div}\left(|\nabla u|^{p-2} \nabla u\right)+a(x)|u|^{p-2} u=\lambda f(x)|u|^{p^{*}-2} u .
$$

By regularity results, such as developped in Guedda-Veron [6], Tolksdorf [17], and by the Vazquez strict maximum principle [18], one gets that $u$ belongs to $C^{1, \alpha}(\bar{\Omega})$, for all $\alpha \in(0,1)$ and that $u>0$ in $\Omega$. Furthermore, multiplying the equation by $u$ and integrating over $\Omega$, one can see that $\lambda$ and $\int_{\Omega} f|u|^{p^{*}}$ are positive. Multiplying $u$ by $\lambda^{\frac{N-p}{p^{2}}}$, one obtains a solution of (4).

Lemma 3.2. Let $\Omega$ be a bounded domain of $\mathbb{R}^{N}$, $p$ a real strictly larger than 1 , and $\left(u_{\epsilon}\right)$ a sequence of $H_{0}^{1}(\Omega)$. We assume that:

1. $\sqrt{\epsilon} u_{\epsilon} \longrightarrow 0$ strongly in $H_{0}^{1}(\Omega)$.

2. $\left(u_{\epsilon}\right)_{\epsilon>0}$ is bounded in $W_{0}^{1, p}(\Omega)$.

3. $\left(\operatorname{div}\left(\frac{\epsilon}{p} \nabla u_{\epsilon}+\left|\nabla u_{\epsilon}\right|^{p-2} \nabla u_{\epsilon}\right)\right)_{\epsilon>0}$ is bounded in $L^{1}(\Omega)$.

Then, there exists $u \in W_{0}^{1, p}(\Omega)$ such that, up to a subsequence, $\left(u_{\epsilon}\right)_{\epsilon>0}$ converges a.e. to $u,\left(\nabla u_{\epsilon}\right)_{\epsilon>0}$ converges a.e. to $\nabla u,\left(\left|\nabla u_{\epsilon}\right|^{p-2} \nabla u_{\epsilon}\right)_{\epsilon>0}$ converges a.e. and weakly in $L^{p^{\prime}}(\Omega)$ to $|\nabla u|^{p-2} \nabla u$ (In fact, $\left(\nabla u_{\epsilon}\right)$ converges strongly to $\nabla u$ in $L^{q}(\Omega)$, for all $\left.q<p\right)$.

Proof. The proof is based on Evans ([9] Th. 3, Chap. 4) and Courilleau-Demengel's arguments ([5] Prop. 3.1). From the boundedness in $L^{p^{\prime}}(\Omega)$ of $\left(\left|\nabla u_{\epsilon}\right|^{p-2} \nabla u_{\epsilon}\right)$, one obtains that, up to a subsequence,

- $\sqrt{\epsilon} \nabla u_{\epsilon} \longrightarrow 0$ a.e. in $\Omega$; 
- $u_{\epsilon} \longrightarrow u$ a.e. in $\Omega$;

- $\Sigma_{\epsilon}=\left|\nabla u_{\epsilon}\right|^{p-2} \nabla u_{\epsilon} \rightarrow \Sigma$ weakly in $L^{p^{\prime}}(\Omega)$;

- $u_{\epsilon} \rightarrow u$ weakly in $W_{0}^{1, p}(\Omega)$.

Let $\delta>0$ be given. By Egoroff's theorem, there exists a universally measurable set $E_{\delta} \subset \subset \Omega$ such that meas $\left(\Omega \backslash E_{\delta}\right)<\delta$, and $u_{\epsilon}$ (respectively $\sqrt{\epsilon} \nabla u_{\epsilon}$ ) tends to $u$ (respectively 0 ) uniformly in $E_{\delta}$. This implies in particular that $\sqrt{\epsilon} \nabla u_{\epsilon} \longrightarrow 0$ strongly in $L^{q}\left(E_{\delta}\right)$, for all $q \leq \infty$.

Now, let $\eta>0$ be given. By the uniform convergence in $E_{\delta}$, there exists $\epsilon_{0}>0$ such that

$$
\left(\epsilon<\epsilon_{0} \quad \Longrightarrow \quad \forall x \in E_{\delta},\left|u_{\epsilon}(x)-u(x)\right|<\frac{\eta}{2}\right) .
$$

Let us consider the following cut-off function $\beta_{\eta}$ :

$$
\beta_{\eta}(t)=\left\{\begin{array}{lll}
t & \text { if } & |t| \leq \eta \\
\eta \frac{t}{|t|} & \text { if } & |t|>\eta
\end{array}\right.
$$

and define $\widetilde{\Sigma}=|\nabla u|^{p-2} \nabla u\left(\widetilde{\Sigma} \in L^{p^{\prime}}(\Omega)\right)$. Since $\beta_{\eta}$ is piecewise $C^{1}$ and continuous, one has that $\beta_{\eta} \circ\left(u_{\epsilon}-u\right)$ $\in W^{1, p}(\Omega)$. Furthermore, one can easily see that

$$
\left(\Sigma_{\epsilon}-\widetilde{\Sigma}\right) \cdot \nabla\left(\beta_{\eta} \circ\left(u_{\epsilon}-u\right)\right) \geq 0 \text { dans } \Omega,
$$

since $\nabla\left(\beta_{\eta} \circ\left(u_{\epsilon}-u\right)\right)=\nabla\left(u_{\epsilon}-u\right)$ for $\epsilon<\epsilon_{0}$ in $E_{\delta}$. Now consider the integral

$$
\int_{E_{\delta}}\left(\left(\Sigma_{\epsilon}-\widetilde{\Sigma}\right) \cdot \nabla\left(u_{\epsilon}-u\right)\right)(x) d x=\int_{E_{\delta}}\left(\left(\frac{\epsilon}{p} \nabla u_{\epsilon}+\Sigma_{\epsilon}-\widetilde{\Sigma}\right) \cdot \nabla\left(u_{\epsilon}-u\right)\right)(x) d x-\int_{E_{\delta}} \frac{\epsilon}{p}\left(\nabla u_{\epsilon} \cdot \nabla\left(u_{\epsilon}-u\right)\right)(x) d x .
$$

Writing the second integral on the right hand side as

$$
-\frac{\epsilon}{p} \int_{E_{\delta}}\left(\nabla u_{\epsilon} \cdot \nabla\left(u_{\epsilon}-u\right)\right)(x) d x=\frac{1}{p}\left(-\left\|\sqrt{\epsilon} u_{\epsilon}\right\|_{L^{2}\left(E_{\delta}\right)}^{2}+\int_{E_{\delta}} \epsilon\left(\nabla u_{\epsilon} \cdot \nabla u\right)(x) d x\right)
$$

one sees that it goes to 0 when $\epsilon$ goes to 0 , since $\sqrt{\epsilon} u_{\epsilon}$ converges to 0 in $L^{p^{\prime}}\left(E_{\delta}\right)$ and in $L^{2}\left(E_{\delta}\right)$. Now, let us treat the first integral as follows:

$$
\begin{aligned}
\int_{E_{\delta}}\left(\left(\frac{\epsilon}{p} \nabla u_{\epsilon}+\Sigma_{\epsilon}-\widetilde{\Sigma}\right) \cdot \nabla\left(u_{\epsilon}-u\right)\right)(x) d x= & \int_{\Omega}\left(\left(\frac{\epsilon}{p} \nabla u_{\epsilon}+\Sigma_{\epsilon}-\widetilde{\Sigma}\right) \cdot \nabla\left(\beta_{\eta} \circ\left(u_{\epsilon}-u\right)\right)\right)(x) d x \\
= & -\int_{\Omega}\left(\left(\operatorname{div}\left(\frac{\epsilon}{p} \nabla u_{\epsilon}+\Sigma_{\epsilon}\right)\right)\left(\beta_{\eta} \circ\left(u_{\epsilon}-u\right)\right)\right)(x) d x \\
& -\int_{\Omega}\left(\widetilde{\Sigma} \cdot \nabla\left(\beta_{\eta} \circ\left(u_{\epsilon}-u\right)\right)\right)(x) d x .
\end{aligned}
$$

On the one hand, $\beta_{\eta} \circ\left(u_{\epsilon}-u\right)$ converges weakly in $W_{0}^{1, p}(\Omega)$, hence

$$
\lim _{\epsilon \rightarrow 0} \int_{\Omega}\left(\widetilde{\Sigma} \cdot \nabla\left(\beta_{\eta} \circ\left(u_{\epsilon}-u\right)\right)\right)(x) d x=0 .
$$

On the other hand, by point 3 , there exists $C>0$ such that, for all $\epsilon>0$,

$$
\left|-\int_{\Omega}\left(\operatorname{div}\left(\frac{\epsilon}{p} \nabla u_{\epsilon}+\Sigma_{\epsilon}\right)\left(\beta_{\eta} \circ\left(u_{\epsilon}-u\right)\right)\right)(x) d x\right| \leq \eta \int_{\Omega}\left|\operatorname{div}\left(\frac{\epsilon}{p} \nabla u_{\epsilon}+\Sigma_{\epsilon}\right)(x)\right| d x \leq C \eta .
$$


Consequently, one has that

$$
\lim _{\epsilon \rightarrow 0} \sup \int_{E_{\delta}}\left(\left(\Sigma_{\epsilon}-\widetilde{\Sigma}\right) \cdot \nabla\left(u_{\epsilon}-u\right)\right)(x) d x \leq C \eta
$$

Since this is true for all $\eta$, one gets that $\left(\Sigma_{\epsilon}-\widetilde{\Sigma}\right) \cdot \nabla\left(u_{\epsilon}-u\right)$ converges a.e. to 0 on $E_{\delta}$. Using Lemma 3.3 below, one has that $\left(\nabla u_{\epsilon}\right)$ converges a.e. to $\nabla u$ in $E_{\delta}$, for all $\delta>0$, and $\delta$ being arbitrary, $\nabla u_{\epsilon}$ tends to $\nabla u$ almost everywhere in $\Omega$. This implies in particular that $\Sigma_{\epsilon}$ converges to $\widetilde{\Sigma}$ a.e., and, since $\left(\Sigma_{\epsilon}\right)$ is bounded in $L^{p^{\prime}}(\Omega)$, weakly in $L^{p^{\prime}}(\Omega)$. Finally, $\Sigma=\widetilde{\Sigma}$.

It could be easily derived from Egoroff's theorem that the convergence of $\left(\nabla u_{\epsilon}\right)$ to $\nabla u$ holds also in every $L^{q}(\Omega)$ spaces, for $q<p$.

To complete the proof, we give Lemma 3.3, which may be found in [4].

Lemma 3.3. Let $p$ be in $(1, \infty)$, and let $\left(X_{k}\right)$ be a sequence of $\mathbb{R}^{N}$ and $X \in \mathbb{R}^{N}$, such that

$$
\lim _{k \rightarrow \infty}\left(\left|X_{k}\right|^{p-2} X_{k}-|X|^{p-2} X\right)\left(X_{k}-X\right)=0 .
$$

Then, $\lim _{k \rightarrow \infty} X_{k}=X$.

\subsection{Localisation method}

We have proved in Section 3.2 that if $\left(u_{\epsilon}\right)$ converges weakly in $W_{0}^{1, p}(\Omega)$ to a function $u$ which is non identically zero, then this limit is a positive solution of (4). We denote by (H1) this assumption.

Let (H2) be the following condition:

$$
K(N, p)^{p} \lambda|f(x)|^{1-p / N}<1
$$

at every point $x$ in $\Omega$, where $f(x)>0$.

We prove in this section that (H1) follows from (H2). For that aim, we adapt the isometry-concentration method used by Hebey [10] and Demengel-Hebey [4] (see also [3] and [12]).

In what follows, we assume that $\left(u_{\epsilon}\right)$ converges weakly to 0 in $W_{0}^{1, p}(\Omega)$.

Let $P \in \bar{\Omega}, \delta>0$, and $\eta \in C_{0}^{\infty}\left(\mathbb{R}^{N}\right), 0 \leq \eta \leq 1$, such that:

$$
\eta(x)=\left\{\begin{array}{lll}
1 & \text { if } & x \in B_{P}(\delta / 2) \\
0 & \text { if } & x \notin B_{P}(\delta)
\end{array}\right.
$$

Let us multiply equation (12) by $\eta^{p} u_{\epsilon}$ and integrate over $\Omega$. One obtains

$$
\frac{\epsilon}{p} \int_{\Omega} \nabla u_{\epsilon} \cdot \nabla\left(\eta^{p} u_{\epsilon}\right)+\int_{\Omega}\left(\left|\nabla u_{\epsilon}\right|^{p-2} \nabla u_{\epsilon} \cdot \nabla\left(\eta^{p} u_{\epsilon}\right)+a(x)\left|u_{\epsilon}\right|^{p} \eta^{p}\right)=\mu_{\epsilon} \int_{\Omega} f(x)\left|u_{\epsilon}\right|^{p^{*}} \eta^{p} .
$$

On the one hand,

$$
\frac{\epsilon}{p} \int_{\Omega} \nabla u_{\epsilon} \cdot \nabla\left(\eta^{p} u_{\epsilon}\right)=\frac{\epsilon}{p} \int_{\Omega} \eta^{p}\left|\nabla u_{\epsilon}\right|^{2}+\epsilon \int_{\Omega} \eta^{p-1} u_{\epsilon} \nabla u_{\epsilon} \cdot \nabla \eta=o(1) \quad(\epsilon \rightarrow 0) .
$$

On the other hand,

$$
\int_{\Omega} a(x)\left|u_{\epsilon}\right|^{p} \eta^{p}=o(1) \quad(\epsilon \rightarrow 0)
$$


since $u_{\epsilon} \longrightarrow 0$ in $L^{p}(\Omega)$. Let us now treat the second term in the left-hand side of (14):

$$
\int_{\Omega}\left|\nabla u_{\epsilon}\right|^{p-2} \nabla u_{\epsilon} \cdot \nabla\left(\eta^{p} u_{\epsilon}\right)=\int_{\Omega} \eta^{p}\left|\nabla u_{\epsilon}\right|^{p}+p \int_{\Omega} \eta^{p-1}\left|\nabla u_{\epsilon}\right|^{p-2} u_{\epsilon} \nabla u_{\epsilon} \cdot \nabla \eta .
$$

First, one has

$$
\begin{aligned}
\left.\left|p \int_{\Omega} \eta^{p-1} u_{\epsilon}\right| \nabla u_{\epsilon}\right|^{p-2} \nabla u_{\epsilon} \cdot \nabla \eta \mid & \leq\|\eta\|_{\infty}^{p-1}\|\nabla \eta\|_{\infty} \int_{\Omega} u_{\epsilon}\left|\nabla u_{\epsilon}\right|^{p-1} \\
& \leq\|\eta\|_{\infty}^{p-1}\|\nabla \eta\|_{\infty}\left(\int_{\Omega}\left|u_{\epsilon}\right|^{p}\right)^{1 / p}\left(\int_{\Omega}\left|\nabla u_{\epsilon}\right|^{p}\right)^{1-1 / p}=o(1) \quad(\epsilon \rightarrow 0) .
\end{aligned}
$$

(Here, $K$ denotes a generic positive constant.)

In addition,

$$
\int_{\Omega}\left|\nabla\left(\eta u_{\epsilon}\right)\right|^{p}=\int_{\Omega}\left|\eta \nabla u_{\epsilon}+u_{\epsilon} \nabla \eta\right|^{p} .
$$

Taking $X=u_{\epsilon} \nabla \eta$ and $Y=\eta \nabla u_{\epsilon}$ in the following inequality, valid for vectors in $\mathbb{R}^{N}$ :

$$
\| X+\left.Y\right|^{p}-|Y|^{p}\left|\leq p\left(|X|^{p-1}+|Y|^{p-1}\right)\right| X \mid
$$

one gets

$$
\begin{aligned}
p \int_{\Omega}|X|^{p} & =p \int_{\Omega}\left|u_{\epsilon}\right|^{p}|\nabla \eta|^{p}=o(1) \quad(\epsilon \rightarrow 0) \\
p \int_{\Omega}|Y|^{p-1}|X| & =\left.p \int_{\Omega} \eta^{p-1} u_{\epsilon}|\nabla \eta| \nabla u_{\epsilon}\right|^{p-1}=o(1) \quad(\epsilon \rightarrow 0)
\end{aligned}
$$

by (16). Using (15-18), and (19), one finally gets

$$
\int_{\Omega}\left|\nabla\left(\eta u_{\epsilon}\right)\right|^{p}+o(1)=\int_{\Omega} \mu_{\epsilon} f(x)\left|u_{\epsilon}\right|^{p^{*}} \eta^{p} .
$$

Assume first that $f(P)<0$. Then, by choosing $\delta$ small enough, one has that $f$ is negative on $B_{P}(\delta)$. Hence

$$
\varlimsup_{\epsilon \rightarrow 0} \int_{\Omega}\left|\nabla\left(\eta u_{\epsilon}\right)\right|^{p}=0 .
$$

Assume now that $f(P) \geq 0$. Writing that

$$
\begin{aligned}
\mu_{\epsilon} \int_{\Omega} f(x) u_{\epsilon}^{p^{*}} \eta^{p} & \leq \mu_{\epsilon} \sup _{B_{P}(\delta)}|f|^{p / p^{*}} \int_{\Omega}\left|\eta u_{\epsilon}\right|^{p}|f(x)|^{1-p / p^{*}}\left|u_{\epsilon}\right|^{p^{*}-p} \\
& \leq \mu_{\epsilon} \sup _{B_{P}(\delta)}|f|^{p / p^{*}}\left(\int_{\Omega}\left|\eta u_{\epsilon}\right|^{p^{*}}\right)^{p / p^{*}}\left(\int_{B_{P}(\delta)}\left|f(x) \| u_{\epsilon}\right|^{p^{*}}\right)^{1-p / p^{*}}
\end{aligned}
$$

and using the definition of $K(N, p)$, one has that

$$
\left(\int_{\Omega}\left|\eta u_{\epsilon}\right|^{p^{*}}\right) \leq K(N, p)^{p} \int_{\Omega}\left|\nabla\left(\eta u_{\epsilon}\right)\right|^{p} .
$$


Thus,

$$
\mu_{\epsilon} \int_{\Omega} f(x)\left|u_{\epsilon}\right|^{p^{*}} \eta^{p} \leq \mu_{\epsilon} \sup _{B_{P}(\delta)}|f|^{p / p^{*}} K(N, p)^{p}\left(\int_{B_{P}(\delta)}|f(x)|\left|u_{\epsilon}\right|^{p^{*}}\right)^{1-p / p^{*}} \int_{\Omega}\left|\nabla\left(\eta u_{\epsilon}\right)\right|^{p} .
$$

Now, if $f(P)=0$, by choosing $\delta$ small enough we have

$$
\mu_{\epsilon} \sup _{B_{P}(\delta)}|f|^{p / p^{*}} K(N, p)^{p}\left(\int_{B_{P}(\delta)}|f(x)|\left|u_{\epsilon}\right|^{p^{*}}\right)^{1-p / p^{*}}<1
$$

Hence,

$$
\varlimsup_{e \rightarrow 0} \int_{\Omega}\left|\nabla\left(\eta u_{\epsilon}\right)\right|^{p}=0 .
$$

In the same manner, if $f(P)>0$, one can choose $\delta$ small enough so that $f$ is positive in $B_{P}(\delta)$. Now, assuming that

$$
\lambda K(N, p)^{p} f(P)^{1-N / p} \varlimsup_{\epsilon \rightarrow 0}\left(\int_{B_{P}(\delta)}|f(x)|\left|u_{\epsilon}\right|^{p^{*}}\right)^{1-p / p^{*}}<1
$$

once more we get

$$
\varlimsup_{\epsilon \rightarrow 0} \int_{\Omega}\left|\nabla\left(\eta u_{\epsilon}\right)\right|^{p}=0
$$

We have obtained the following result

Lemma 3.4. Assume that (H1) does not hold and that, for every point $P$ in $\bar{\Omega}$ such that $f(P)>0$, there exists some real $\delta_{P}>0$ satisfying

$$
\lambda K(N, p)^{p} f(P)^{1-p / N} \varlimsup_{\epsilon \rightarrow 0}\left(\int_{B_{P}\left(\delta_{P}\right)}\left|f(x) \| u_{\epsilon}\right|^{p^{*}}\right)^{1-p / p^{*}}<1 .
$$

Then,

$$
\lim _{\epsilon \rightarrow 0} \int_{\Omega}\left|\nabla\left(\eta_{P} u_{\epsilon}\right)\right|^{p}=0
$$

where $\eta_{P}$ denotes the cut-off function defined at the beginning of the section.

We can now prove that (H1) follows from (H2). According to the assumptions of Lemma 3.4 , for all $x \in \bar{\Omega}$, there exists $\delta_{x}>0$, and a cut-off function $\eta_{x}$, verifying

$$
\lim _{\epsilon \rightarrow 0} \int_{\Omega}\left|\nabla\left(\eta_{x} u_{\epsilon}\right)\right|^{p}=0
$$

By the compactness of $\bar{\Omega}$, one can find a finite number of points $\left(x_{i}\right)_{1 \leq i \leq k}$ and reals $\left(\delta_{x_{i}}\right)_{1 \leq i \leq k}$ such that

$$
\bar{\Omega}=\bigcup_{i=1}^{k} B_{x_{i}}\left(\delta_{x_{i}}\right)
$$


By convexity, one gets

$$
\int_{\Omega}\left|\nabla u_{\epsilon}\right|^{p} \leq k^{p-1} \sum_{i=1}^{k} \int_{\Omega}\left|\nabla\left(\eta_{x_{i}} u_{\epsilon}\right)\right|^{p}
$$

thus,

$$
\lim _{\epsilon \rightarrow 0} \int_{\Omega}\left|\nabla u_{\epsilon}\right|^{p}=0
$$

which contradicts $u_{\epsilon} \in \mathcal{H}_{p^{*}}$, since $\int_{\Omega} f\left|u_{\epsilon}\right|^{p^{*}}=1, \forall \epsilon>0$.

Consequently, there exists $P \in \bar{\Omega}$, such that $f(P)>0$, which verifies

$$
\forall \delta>0, \quad K(N, p)^{p} \lambda f(P)^{p / p^{*}} \varlimsup_{\epsilon \rightarrow 0}\left(\int_{B_{P}(\delta)}|f(x)|\left|u_{\epsilon}\right|^{p^{*}}\right)^{1-p / p^{*}} \geq 1 .
$$

Furthermore, one can choose $\delta$ small enough in order to have

$$
1 \geq \int_{B_{P}(\delta)}|f(x)|\left|u_{\epsilon}\right|^{p^{*}}+\int_{f \leq 0} f(x)\left|u_{\epsilon}\right|^{p^{*}} .
$$

The previous computations proves that $\left(u_{\epsilon}\right)$ converges strongly in $L^{p^{*}}$ in the neighbourhood of every point where $f$ is nonnegative, and then, one obtains that

$$
\varlimsup_{\epsilon \rightarrow 0} \int_{B_{P}(\delta)}|f(x)|\left|u_{\epsilon}\right|^{p^{*}} \leq 1 .
$$

This contradicts (H2), and then Lemma 3.4 implies that the limit $u$ cannot be identically zero. To complete the proof of Theorem 2.2, it remains to show that (a subsequence of) $\left(u_{\epsilon}\right)$ converges strongly to a solution $u$.

Proposition 3.6. Up to a subsequence, $\left(u_{\epsilon}\right)$ converges strongly to $u$ in $W_{0}^{1, p}(\Omega)$ as $\epsilon$ tends to 0 .

Proof. We have proved before that every subsequence of $\left(u_{\epsilon}\right)$ which converges weakly in $W_{0}^{1, p}(\Omega)$ has a limit $\mathrm{u}$ which is positive in $\Omega$. Let us show that, in fact, the convergence is strong. For that aim, it is sufficient to show that the $L^{p}$-norm of the gradient $\left(\nabla u_{\epsilon}\right)$ converge to $\|\nabla u\|_{L^{p}}$. According to Proposition 3.5, $u$ is a solution of

$$
-\operatorname{div}\left(|\nabla u|^{p-2} \nabla u\right)+a(x)|u|^{p-2} u=\lambda f(x)|u|^{p^{*}-2} u
$$

then, multiplying by $u$ and using Green's formula, one gets

$$
\int_{\Omega}\left(|\nabla u|^{p}+a(x)|u|^{p}\right)=\lambda \int_{\Omega} f(x)|u|^{p^{*}} .
$$

First, let us prove that $\lambda=J_{0}(u)$.

Set $v=\left(\int f(x)|u|^{p^{*}}\right)^{-1 / p^{*}} u$. The equation above writes

$$
\left(\int_{\Omega} f(x)|u|^{p^{*}}\right)^{p / p^{*}} \int_{\Omega}\left(|\nabla v|^{p}+a(x)|v|^{p}\right)=\lambda \int_{\Omega} f(x)|u|^{p^{*}} .
$$


Since $v \in \mathcal{W}_{p^{*}}, J_{0}(v) \geq \lambda$, and then

$$
\int_{\Omega} f(x)|u|^{p^{*}} \geq 1
$$

Furthermore, since $u_{\epsilon} \longrightarrow u$ in $W_{0}^{1, p}(\Omega)$, one can write

$$
\lambda \int_{\Omega} f(x)|u|^{p^{*}}=\int_{\Omega}\left(|\nabla u|^{p}+a(x)|u|^{p}\right) \leq \lim \inf _{\epsilon \rightarrow 0} \epsilon \int_{\Omega}\left|\nabla u_{\epsilon}\right|^{2}+\int_{\Omega}\left(\left|\nabla u_{\epsilon}\right|^{p}+a(x)\left|u_{\epsilon}\right|^{p}\right) \leq \lim \inf _{\epsilon \rightarrow 0} \lambda_{\epsilon} \leq \lambda
$$

which gives the result. Actually, $J_{\epsilon}(u)$ tends to $J_{0}(u)$, then

$$
\lim _{\epsilon \rightarrow 0} \int_{\Omega}\left|\nabla u_{\epsilon}\right|^{p}=\int_{\Omega}|\nabla u|^{p}
$$

and the proof of Theorem 2.2 is completed.

\section{A COntinuity Result}

In this section, we are interested in the continuity of the set of the solutions for equation (4). Here, we prove that $u \equiv 0$ is isolated among the solutions of (4), in some sense which will be precised later. First, we give a result concerning the isolation of 0 in a strong sense.

Lemma 4.1. Let $u \in W_{0}^{1, p}(\Omega)$ be a solution of (4).

- If $\int_{\Omega} f(x)|u|^{p^{*}}=0$, then $u \equiv 0$.

- If this integral is not zero, then we have $\int_{\Omega} f(x)|u|^{p^{*}} \geq \lambda^{N / p}$.

Proof. Suppose that $\int_{\Omega} f(x)|u|^{p^{*}}=0$, multiply by $u$ and integrate over $\Omega$, to obtain

$$
J(u)=\int_{\Omega}\left(|\nabla u|^{p}+a(x)|u|^{p}\right)=0
$$

thus $u \equiv 0$ by coercivity of $L$.

Suppose now that $u \not \equiv 0$, so that $\int_{\Omega} f(x)|u|^{p^{*}}>0$. Defining

$$
w=\left(\int_{\Omega} f(x)|u|^{p^{*}}\right)^{-1 / p^{*}} u
$$

one has, by construction, that $w \in \mathcal{W}_{p^{*}}(G)$, hence

$$
\lambda \leq\left(\int_{\Omega} f(x)|u|^{p^{*}}\right)^{-p / p^{*}} J(u) \leq\left(\int_{\Omega} f(x)|u|^{p^{*}}\right)^{1-p / p^{*}}
$$

and actually

$$
\int_{\Omega} f(x)|u|^{p^{*}} \geq \lambda^{N / p}
$$


Remark. Here, one can see that $\lambda$ represents a minimal energy level for non zero solutions of (4).

Now, we prove a stronger result which prevents, under some assumption on the energy level of considered solutions, from concentration phenomena that could happen if the sequence converges weakly but not strongly in $W_{0}^{1, p}(\Omega)$.

In what follows, we assume that the existence's condition of Theorem 2.2 holds, that is on every point $x \in \bar{\Omega}$ where $f(x)>0$,

$$
\lambda f(x)^{1-p / N} K(N, p)^{p}<1
$$

Theorem 4.1. Let $\left(u_{n}\right)$ be a bounded sequence (in $W_{0}^{1, p}(\Omega)$ ) of solutions of (4) which are non identically zero. We assume that

$$
\lim _{n \rightarrow \infty} \int_{\Omega} f(x)\left|u_{n}\right|^{p^{*}}=\lambda^{N / p}
$$

Then, if $\left(u_{n}\right)$ converges weakly to $u, u$ is a non identically zero solution of (4) (In fact, $\left(u_{n}\right)$ converges strongly to $u$ in $W^{1, p}(\Omega)$.)

Remark. This result can be extended to positive and nodal solutions, when the data $\Omega, a$ and $f$ present some symmetries.

Proof. The fact that $u$ is a solution of (4) immediately follows from Section 3.2. It is sufficient to show that $u$ is not the trivial function.

For that aim, we use the localisation method in Section 3.3. Let $P \in \bar{\Omega}, \delta$ be a positive real, and $\eta$ be the cut-off function defined in Section 3.3. Following the proof of Lemma 3.4, and assuming that $\left(u_{n}\right) \rightarrow 0$ in $W_{0}^{1, p}(\Omega)$, one obtains that

$$
\int_{\Omega}\left|\nabla\left(\eta u_{n}\right)\right|^{p}+o(1)=\int_{\Omega} f(x)\left|u_{n}\right|^{p^{*}} \eta^{p} \quad(n \rightarrow \infty)
$$

- If $f(P)<0$, one has, for $\delta$ small enough, that $\int_{\Omega}\left|\nabla\left(\eta u_{n}\right)\right|^{p}$ tends to 0 as $n$ tends to $\infty$.

- If $f(P) \geq 0$, one has the following estimate:

$$
\left.\int_{\Omega} f(x)\left|u_{n}\right|^{p^{*}} \eta^{p} \leq \sup _{B_{P}(\delta)}|f|^{p / p^{*}} K(N, p)^{p}\left(\int_{B_{P}(\delta)}|f(x)|\left|u_{n}\right|^{p^{*}}\right)^{1-p / p^{*}} \int_{\Omega}|\nabla| \eta u_{n}\right)\left.\right|^{p} .
$$

Hence,

* for $f(P)=0$, the conclusion is the same;

* for $f(P)>0$, one assumes that

$$
K(N, p)^{p} f(P)^{1-p / N} \varlimsup_{n \rightarrow \infty}\left(\int_{B_{P}(\delta)}|f(x)|\left|u_{n}\right|^{p^{*}}\right)^{1-p / p^{*}}<1
$$

which yields that

$$
\lim _{n \rightarrow \infty} \int_{\Omega}\left|\nabla\left(\eta u_{n}\right)\right|^{p}=0
$$


Then, if (20) holds at every point $P$ where $f(P)>0$, one has by compactness that $u_{n} \longrightarrow 0$ in $W_{0}^{1, p}(\Omega)$, which is a contradiction with the estimate

$$
\int_{\Omega} f(x)\left|u_{n}\right|^{p^{*}} \geq \lambda^{N / p}>0 .
$$

It follows that there exists some $P \in \bar{\Omega}$ such that $F(P)>0$ and

$$
K(N, p)^{p} f(P)^{1-p / N} \varlimsup_{n \rightarrow \infty}\left(\int_{B_{P}(\delta)}\left|f(x) \| u_{n}\right|^{p^{*}}\right)^{p / N} \geq 1
$$

and then, by the condition in Theorem 2.2 ,

$$
\varlimsup_{n \rightarrow \infty}\left(\int_{B_{P}(\delta)}|f(x)|\left|u_{n}\right|^{p^{*}}\right)^{p / N}>\lambda .
$$

Noting that for $\delta$ small enough,

$$
\int_{\Omega} f(x)\left|u_{n}\right|^{p^{*}} \geq \int_{B_{P}(\delta)}|f(x)|\left|u_{n}\right|^{p^{*}}+\int_{f \leq 0} f(x)\left|u_{n}\right|^{p^{*}}
$$

and using the fact that the second integral of the right-hand side tends to 0 , and the left-hand side to $\lambda^{N / p}$, one obtains

$$
\lambda \geq \varlimsup_{n \rightarrow \infty}\left(\int_{B_{P}(\delta)}|f(x)|\left|u_{n}\right|^{p^{*}}\right)^{p / N}
$$

which is a contradiction with (21).

Finally, with the same arguments as in Proposition 3.6, one obtains the strong convergence of the sequence $\left(u_{n}\right)$ to $u$, which completes the proof.

\section{THE CASE WHERE $\Omega$ IS INVARIANT UNDER SYMMETRIES}

This section is devoted to the case where the domain $\Omega$ is invariant under the action of some subgroup $G$ of the orthogonal group $O_{N}(\mathbb{R})$. We give the analogous stability results for positive and nodal extremal functions (a function is said to be nodal if it changes sign).

Let $G$ be a subgroup of $O_{N}(\mathbb{R})$ and $\sigma$ be an involution of $O_{N}(\mathbb{R}), \sigma \neq I d$. We assume that $G$ is compact (if it is not the case, one may take the closure $\bar{G}$ of $G$, since every $G$-invariant function is $\bar{G}$-invariant) Let us also define the sets

$$
\begin{gathered}
\mathcal{W}_{p^{*}}(G)=\left\{v \in W_{0}^{1, p}(\Omega) ; \forall \tau \in G, v \circ \tau=v, \int_{\Omega} f(x)|v|^{p^{*}}=1\right\}, \\
\mathcal{W}_{p^{*}}^{\sigma}(G)=\left\{v \in W_{0}^{1, p}(\Omega) ; \forall \tau \in G, v \circ \tau=v, v \circ \sigma=-v, \int_{\Omega} f(x)|v|^{p^{*}}=1\right\}, \\
\mathcal{W}_{p^{*}}(G)=\mathcal{H}_{p^{*}}(G) \cap W_{0}^{1, p}(\Omega), \quad \mathcal{W}_{p^{*}}^{\sigma}(G)=\mathcal{H}_{p^{*}}^{\sigma}(G) \cap W_{0}^{1, p}(\Omega),
\end{gathered}
$$


and, for $\epsilon \geq 0$, the quantities

$$
\begin{aligned}
& \lambda_{\epsilon}(G)=\inf _{v \in \mathcal{H}_{p^{*}}(G)} J_{\epsilon}(v), \\
& \lambda_{\epsilon}^{\sigma}(G)=\inf _{v \in \mathcal{H}_{p^{*}}^{\sigma}(G)} J_{\epsilon}(v) .
\end{aligned}
$$

Finally, one defines

$$
\begin{gathered}
\lambda(G)=\inf _{v \in \mathcal{W}_{p^{*}}(G)} J_{0}(v), \\
\lambda^{\sigma}(G)=\inf _{v \in \mathcal{W}_{p^{*}}^{\sigma}(G)} J_{0}(v) .
\end{gathered}
$$

Arguing as in the proof of Lemma 3.1, one can show that $\lambda_{0}(G)=\lambda(G)$ and $\lambda_{0}^{\sigma}(G)=\lambda^{\sigma}(G)$.

Then, the results are the following:

Theorem 5.1. We assume that $\Omega$, a, and $f$ are $G$-invariant and that, for every point $x \in \bar{\Omega}$ such that $f(x)>0$,

$$
\lambda(G) f(x)^{1-\frac{p}{N}} K(N, p)^{p}<\left(\operatorname{Card} O_{G}(x)\right)^{\frac{p}{N}} .
$$

Then,

1) for every $\epsilon>0$, there exists a nonnegative function $u_{\epsilon} \in \mathcal{H}_{p^{*}}(G)$ which realizes the infimum in (22). In addition, $u_{\epsilon}$ is a weak solution of the following equation:

$$
-\frac{\epsilon}{p} \Delta u_{\epsilon}-\operatorname{div}\left(\left|\nabla u_{\epsilon}\right|^{p-2} \nabla u_{\epsilon}\right)+a(x)\left|u_{\epsilon}\right|^{p-2} u_{\epsilon}=\mu_{\epsilon}(G) f(x)\left|u_{\epsilon}\right|^{p^{*}-2} u_{\epsilon}
$$

where

$$
\mu_{\epsilon}(G)=\lambda_{\epsilon}(G)+\epsilon\left(\frac{1}{p}-\frac{1}{2}\right) \int_{\Omega}\left|\nabla u_{\epsilon}\right|^{2}
$$

2) Up to a subsequence, $\left(u_{\epsilon}\right)$ converges strongly in $W_{0}^{1, p}(\Omega)$ to a positive and $G$-invariant solution $u$ of equation (4). Furthermore, $u$ is an extremal function for (24).

Theorem 5.2. We assume that $\Omega$, a, and $f$ are $H$-invariant. Suppose that

$$
\exists x \in \bar{\Omega} ; f(x)>0 \text { and } \sigma\left(O_{G}(x)\right) \cap O_{G}(x)=\emptyset
$$

and that, for every $x \in \bar{\Omega}$, verifying $f(x)>0$,

$$
\lambda^{\sigma}(G) f(x)^{1-\frac{p}{n}} K(N, p)^{p}<\left(\operatorname{Card} O_{H}(x)\right)^{\frac{p}{N}} .
$$

Then, we have

1) for every $\epsilon>0$, there exists a function $u_{\epsilon} \in \mathcal{H}_{p^{*}}^{\sigma}(G)$ which realizes the infimum in (23). In addition, $u_{\epsilon}$ is a weak solution of the equation:

$$
-\epsilon \Delta u_{\epsilon}-\operatorname{div}\left(\left|\nabla u_{\epsilon}\right|^{p-2} \nabla u_{\epsilon}\right)+a(x)\left|u_{\epsilon}\right|^{p-2} u_{\epsilon}=\mu_{\epsilon}^{\sigma}(G) f(x)\left|u_{\epsilon}\right|^{p^{*}-2} u_{\epsilon}
$$


where

$$
\mu_{\epsilon}^{\sigma}(G)=\lambda_{\epsilon}^{\sigma}(G)+\epsilon\left(\frac{1}{p}-\frac{1}{2}\right) \int_{\Omega}\left|\nabla u_{\epsilon}\right|^{2}
$$

2) Up to a subsequence, $\left(u_{\epsilon}\right)$ coverges strongly in $W_{0}^{1, p}(\Omega)$ to a $G$-invariant and $\sigma$-antisymmetrical solution $u$ of equation (4). Furthermore, $u$ is an extremal function for (25).

The assumption (28) provides that the set $\mathcal{W}_{p^{*}}^{\sigma}(G)$ is not empty. For Theorems 5.1 and 5.2 , all the arguments developped in Section 3 remain valid, under the condition that there exists a symmetrization of functions for each problem, which leaves the functionnals invariant. The symmetrizations are the following: for a function $v$, one takes

$$
v_{G}(x)=\int_{G} v \circ \tau d \mu(\tau)
$$

where $\mu$ is the Haar measure of $G$. (It exists since $G$ is locally compact.) For the problem of nodal solutions, one takes

$$
v_{G}^{\sigma}(x)=v_{G} \circ \sigma(x)-v_{G}(x)
$$

with $v_{G}$ defined as above.

I want to thank the referees for the remarks they did about the first version of this paper.

\section{REFERENCES}

[1] T. Aubin, Problèmes isopérimétriques et espaces de Sobolev. J. Differential Geom. 11 (1976) 573-598.

[2] T. Aubin, Nonlinear Analysis on Manifolds. Monge-Ampere equations, Springer-Verlag (1982) (Grundlehren) 252.

[3] O. Druet, Generalized scalar curvature type equations on compact riemaniann manifolds. Preprint of the University of CergyPontoise (1997).

[4] F. Demengel and E. Hebey, On some nonlinear equations involving the $p$-Laplacian with critical Sobolev growth. Adv. in $P D E$ 's, to appear.

[5] P. Courilleau and F. Demengel, On the heat flow for $p$-harmonic maps with values in $S^{1}$. Nonlinear Anal. TMA, accepted.

[6] M. Guedda and L. Veron, Local and global properties of solutions of quasilinear elliptic equations. J. Differential Equations 76 (1988) 159-189.

[7] M. Guedda and L. Veron, Quasilinear elliptic equations involving critical Sobolev exponents. Nonlinear Analysis, Theory, Methods and Applications 13 (1989) 879-902.

[8] E. Hebey and M. Vaugon, Existence and multiplicity of nodal solutions for nonlinear elliptic equations with critical Sobolev growth. J. Funct. Anal. 119 (1994) 298-318.

[9] L.C. Evans, Weak convergence methods for nonlinear partial differential equations. Conference Board of the Mathematical Sciences 74 (1990).

[10] E. Hebey, La méthode d'isométries-concentration dans le cas d'un problème non linéaire sur les variétés compactes à bord avec exposant critique de sobolev. Bull. Sci. Math. 116 (1992) 35-51.

[11] E. Hebey, Sobolev Spaces on Riemannian Manifolds, Springer-Verlag (1996) (LNM) 1635.

[12] A. Jourdain, Solutions nodales pour des equations de type courbure scalaire sur la sphère. Preprint of the University of Cergy-Pontoise (1997).

[13] P.L. Lions, The concentration-compactness principle in the calculus of variations. The limit case, part I. Revista Matematica Iberoamericana 1 (1985) 145-199.

[14] P.L. Lions, The concentration-compactness principle in the calculus of variations. The limit case, part II. Revista Matematica Iberoamericana 1 (1985) 45-116.

[15] B. Nazaret, Stabilité sous des perturbations visqueuses des solutions d'équations du type $p$-Laplacien avec exposant critique de Sobolev. Preprint of the University of Cergy-Pontoise (5/98).

[16] G. Talenti. Best constants in Sobolev inequalities. Ann. Mat. Pura Appl. 110 (1976) 353-372.

[17] P. Tolksdorf, Regularity for a more general class of quasilinear elliptic equations. J. Differential Equations 51 (1984) $126-150$.

[18] J.L. Vazquez, A strong maximum principle for some quasilinear elliptic equations. Appl. Math. Optim. 12 (1984) 191-202. 